\title{
Proposed Model of Electric Field Effects in High-Purity GaAs at Room Temperature
}

\author{
Durga Prasad Sapkota1 ${ }^{*}$, Madhu Sudan Kayastha², Makoto Takahashi' ${ }^{2}$ Koichi Wakita ${ }^{2}$ \\ ${ }^{1}$ Research and Development, Nepal Telecom, Kathmandu, Nepal \\ ${ }^{2}$ Graduate School of Engineering, Chubu University, Kasugai, Japan \\ Email: ${ }^{*}$ durga.sapkota@ntc.net.np
}

Received 3 April 2014; revised 1 May 2014; accepted 21 May 2014

Copyright (C) 2014 by authors and Scientific Research Publishing Inc.

This work is licensed under the Creative Commons Attribution International License (CC BY).

http://creativecommons.org/licenses/by/4.0/

(c) (i) Open Access

\begin{abstract}
We have proposed a new model for the calculation of excitonic electroabsorption based on modified previously reported models for bulk structure. The excitonic absorption spectra in high purity GaAs have been theoretically studied in the presence of electric field at room temperature (RT). The Stark shift, linewidth broadening of exciton and extinction ratio have been calculated as a function of electric field. For the validity of our model we have compared with experimental result.
\end{abstract}

\section{Keywords}

Optoelectronics, Electroabsorption, Modulator

\section{Introduction}

Electroabsorption modulators are semiconductor devices which can be used for controlling the intensity of a laser beam via an electric voltage and have only been reported for waveguide configuration. Surface normal mode results in low extinction ratio due to short depletion width. We succeeded in growing high-purity epitaxial layers $\left(\sim 1 \times 10^{13} \mathrm{~cm}^{-3}\right)$ and obtaining high extinction ratio over $20 \mathrm{~dB}$ [1]. Based on the well known model [2] [3] (Franz-Keldysh effect) such large extinction ratio cannot be explained. We also observed clear exciton peak [4] and large red-shift associated with applying voltage as reported for quantum well structures. Therefore we propose a new model based on the experimental data.

Super-parallel and high speed operation that is characteristic of light can be realized by spatial light modulators (SLMs). However, at present a liquid-crystal light valve is only practically used with a low contrast ratio and low speed. Surface normal electroabsorption modulators are expected to be a next-generation SLMs with high speed and highly efficient SLMs.

${ }^{*}$ Corresponding author. 
The theoretical and experimental results have been reported [5]-[8]. Casey et al. [9] has discussed the strong relationship between the background impurities and optical absorption in bulk GaAs material. They pointed out that the contribution of exciton on absorption can be observed in high purity $\left(5 \times 10^{16} \mathrm{~cm}^{-3}\right)$ bulk GaAs even at RT. Dow and Redfield have theoretically studied about the importance of excitonic effects on electroabsorption [10]. The experimental result of high-purity GaAs epilayers has been succeeded with background impurity less than $1 \times 10^{13} \mathrm{~cm}^{-3}$ at RT and Hall mobility of $312,000 \mathrm{~cm}^{2} \mathrm{~V}^{-1} \cdot \mathrm{s}^{-1}$ at $77 \mathrm{~K}$ by using a liquid phase epitaxy (LPE) method [4]. A clear exciton absorption peak at RT is observed in this high purity epilayer which makes it possible to develop a spatial light modulator (SLM). This epilayer with large depletion layer over $30 \mu \mathrm{m}$ is operated at surface normal configuration.

The experimental and theoretical study on excitonic electroabsorption in high purity GaAs at RT was realized [11], where F-K broadening has been used as Bottka et al. [7] and Sommerfeld factor was also not considered. In this work, we have proposed a new model for the calculation of excitonic electro absorption based on previously reported models. The Stark red-shifts, linewidth broadening of exciton and extinction ratio have been calculated. A new SLM with high speed and low driving voltage can be proposed by using these results. For the validity of our model we have compared with experimental result [12] and found the close agreement between them.

\section{Theoretical Models}

The absorption coefficient with exciton band and continuum band transition including Gaussian function and Sommerfeld factor at RT can be generalized based on [13] for bulk heavy hole only

$$
\begin{gathered}
\alpha(\hbar \omega)=\alpha_{g}\left(\frac{n_{r} E_{g}}{\hbar \omega}\right)\left[\alpha_{e}(x)+\alpha_{c}(x)\right] \\
\text { where } \alpha_{e}(x)=G(x) \text { and } \alpha_{c}(x)=H(x)+S(x) \\
x=\frac{\left(\hbar \omega-E_{e x}\right)}{\hbar \Gamma} \\
G(x)=Q_{X} e^{-\frac{x^{2}}{2}} \\
H(x)=\frac{1}{1+e^{x}} \\
S(x)=\frac{1}{1+e^{-2 \pi x}} \\
\alpha_{g}=\left[\frac{\left|M_{c v}\right|}{\hbar k_{g o} n_{r}}\right]^{2}\left[\frac{\left(4 \pi C_{F}\right)^{2}}{\varepsilon_{s}}\right]\left[\frac{\left(m_{r}\right)^{2}}{3\left(m_{0}\right)^{2}}\right]\left(\frac{n_{r}}{\lambda_{g 0}}\right) \\
C_{F}=\frac{2 \pi \mu_{0} c_{0} e^{2}}{2 \hbar}
\end{gathered}
$$

where $n_{r}$ is refractive index at bandgap, $\hbar \omega$ is photon energy, $E_{\text {ex }}$ is exciton transition energy, $c_{0}$ is velocity of light, $\varepsilon_{\mathrm{s}}$ is static dielectric constant, $\mu_{0}$ is vacuum permeability, $M_{c v}$ is dipole matrix element, $k_{g 0}=2 \pi / \lambda_{g}$, where $\lambda_{g 0}$ is free space bandgap wavelength, e is electron change, $C_{F}$ is fine-structure constant, $m_{r}$ is reduced mass of electron and hole, $m_{0}$ is free electron mass, $G(x)$ is Gaussian function, $Q_{X}$ is exciton quenching factor, $S(x)$ is three dimensional Sommerfeld factor and $H(x)$ is Heaviside function.

\section{Result and Discussion}

Equation (1) is divided into two parts: one is absorption spectra with excitonic transition $\left(\alpha_{e}\right)$ which is represented by Gaussian function and the other is band continuum absorption spectra $\left(\alpha_{c}\right)$ which is represented by Heaviside and Sommerfeld factor. The Sommerfeld factor was considered for fitting with the experimental re- 
sult. The optical matrix element has been calculated as defined in the Ref. 13.

The absorption spectra with excitonic effects for high-purity GaAs with $5 \mu \mathrm{m}$ thick with background impurity of $10^{14} \mathrm{~cm}^{-3}$ at various electric fields are shown in Figure 1. The Stark red-shift of exciton peak with and with out external applied voltage has been included as $\Delta E=-6 \times 10^{-3} \frac{9}{8} f^{2} \mathrm{meV}$ and $\Delta E=-19 \times 10^{-3} \frac{9}{8} f^{2} \mathrm{meV}$ where $f$ is reduced electric field which is defined as $f=F / F_{i}$ where $F_{\mathrm{i}}$ is ionization field and $F$ is electric field, respectively. The inhomogeneous exciton linewidth broadening due to variation of electric field causing from background impurity in intrinsic region is also included according to the [14] by fitting with the experimental results as $\hbar \Gamma=26 \times 6.5 \times 10^{-3} \frac{1}{f} e^{\frac{-10}{3 f}} \mathrm{meV}$.

The electroabsorption spectrum broadening due to continuum band transition (F-K effect) has been included as 2.6 times smaller than that of Bottka et al. [7] for fitting with the experimental result. In zero external voltage, the loss is estimated to be about $0.09 \mathrm{~dB}$ at photon energy of $1.404 \mathrm{eV}$. In our model, we have not considered the reflection loss, coupling loss and substrate loss due to which the absorption spectra are slightly higher in experimental result than that of the theoretical result at photon energy below $1.38 \mathrm{eV}$. In Figure 1 the gap of absorption tail between experimental and theoretical results without external applied voltage starts at photon energy of $1.415 \mathrm{eV}$, on the other hand, the gap with external applied voltage nearly starts at $1.38 \mathrm{eV}$.

The reason behind this is that the contribution of the F-K effect dominates the excitonic effects at high electric field. The electric field induced Stark red-shift of exciton resonance remains stable toward the lower energy even at electric field of $70 \mathrm{kV} / \mathrm{cm}$ indicating that the electric field is uniformly distributed inside the active layer, which predicts very high-purity thin layer. It is fortunate that the large amount of Stark red-shift of 20 meV was obtained, which nearly conceded with the experimental results as shown in Figure 2. The maximum change in absorption coefficient is obtained roughly about $7000 \mathrm{~cm}^{-1}$ at $1.404 \mathrm{eV}$. In bulk GaAs, it is first time reported that the large red-shift and much change in absorption in EA modulation are obtained for surface normal configuration. Up to a field of $25 \mathrm{kV} / \mathrm{cm}$, the shift of exciton resonance follows slightly and then abruptly after this electric field. This is due to the starting of the degeneration of mixing of excitonic band and continuum band at high electric field. At field of $70 \mathrm{kV} / \mathrm{cm}$, the exciton absorption red-shift exceeds to the exciton binding energy experimentally measured as $6.5 \mathrm{meV}$ at zero external applied voltage.

We obtained relatively large value of exciton binding energy and small value of Bohr radius as a result the exciton resonance is slightly broadened up to 14 times of classical ionization field as shown in Figure 3 . In this study, the obtained ionization field is only 1.26 times smaller than GaN, whereas, it was reported about 17 times smaller [15]. We predict that the exciton peak after the electric field of $70 \mathrm{kV} / \mathrm{cm}$ may be completely dominated by $\mathrm{F}-\mathrm{K}$ effect. We have calculated the extinction ratio by using the relation $10 \log \left(e^{-\Delta \alpha d}\right)$ as a function of electric field strength at detuning energy of $25 \mathrm{meV}$. Figure 4 shows that the gap of curve between the extinct-

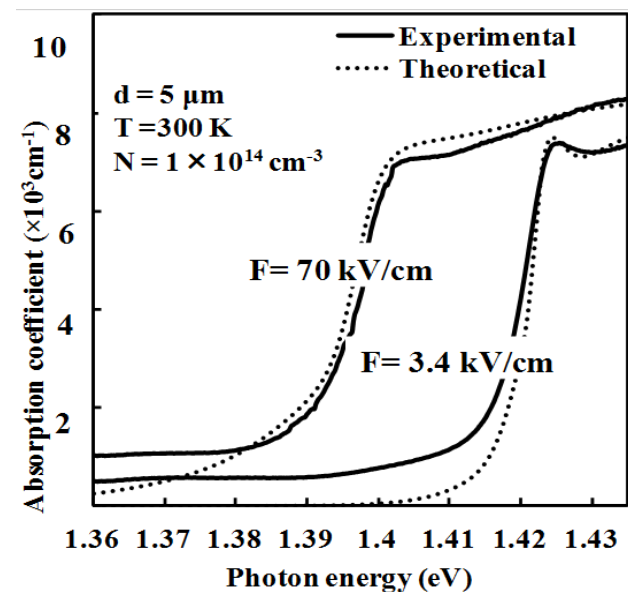

Figure 1. The comparison of excitonic absorption spectra between experimental and theoretical result. 


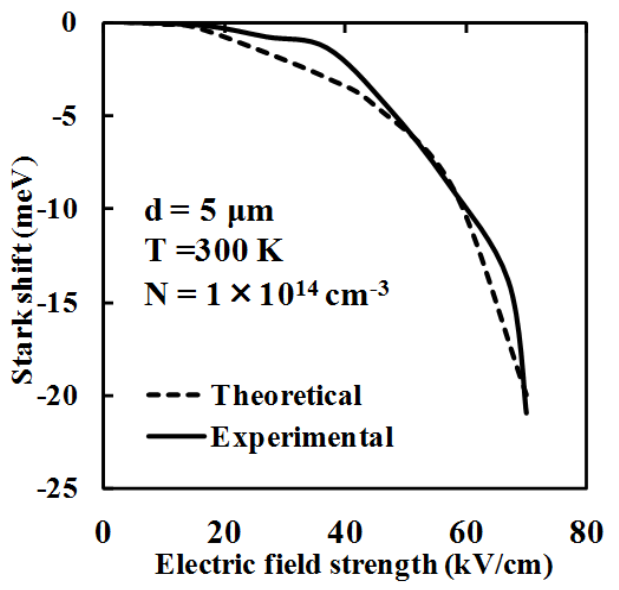

Figure 2. Theoretical and experimental results of Stark red-shift as a function of electric field.

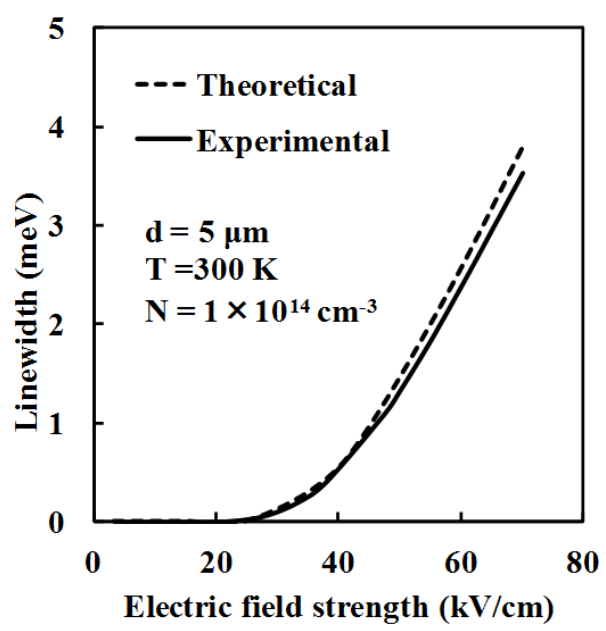

Figure 3. Theoretical and experimental results of linewidth as a function of electric field.

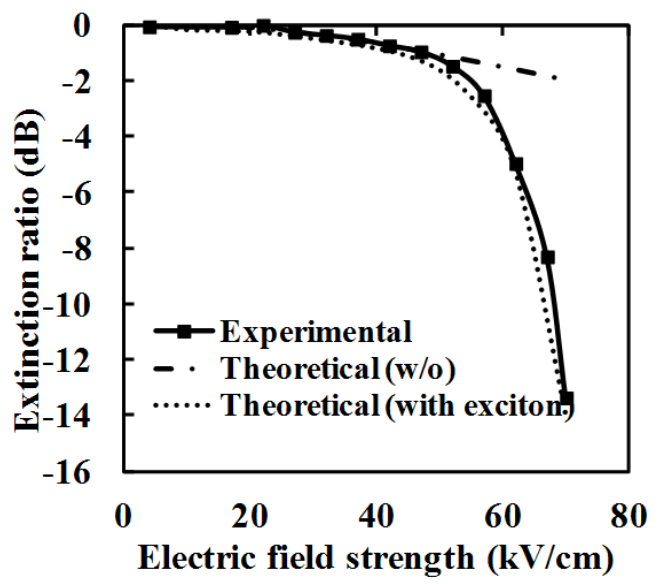

Figure 4. The extinction ratio as a function of electric field strength. The solid line, dot line and dash dot line represent the experimental, theoretical (with exciton) and theoretical (w/o exciton), respectively. 
tion ratio with and without exciton is very small at low electric field. The separation increases with the increasing of electric field. The extinction ratio is estimated to be about 7 times higher in the case of exciton absorption than that of without exciton at $70 \mathrm{kV} / \mathrm{cm}$. This is due to the advantage of enhancement of absorption with exciton as a result; we obtained the large absorption coefficient change in high-purity GaAs material. The extinction ratio is estimated to be $14 \mathrm{~dB}$ for high speed device at $70 \mathrm{kV} / \mathrm{cm}$, however the applied voltage is comparatively high due to relatively large background impurity.

\section{Summary}

We have developed a new model for excitonic electroabsorption spectra, Stark red-shift, and inhomogeneous linewidth broadening in the presence of electric field strength based on previously reported result. We also obtained the large red-shift about 3 times higher than the exciton binding energy without excessive exciton linewidth broadening. This is appreciable result in bulk structure. The performance characteristic is determined to be an extinction ratio of $14 \mathrm{~dB}$.

\section{References}

[1] Kayastha, M.S., Matsunami, I., Sapkota, D.P., Takahashi, M. and Wakita, K. (2009) Ultrahigh-Purity Undoped GaAs Epitaxial Layers Prepared by Liquid Phase Epitaxy. Japanese Journal of Applied Physics, 48, 121102-121106. http://dx.doi.org/10.1143/JJAP.48.121102

[2] Franz, W and Naturforsch, Z. (1958) 13a, 484.

[3] Keldysh, L.V. and Eksperim, Zh. (1958) Teor. Fiz., 34 [Sov. Phys. JETP 7, 788].

[4] Kayastha, M.S., Takahashi, M. and Wakita, K. (2010) High-Extinction Ratio and Low-Driving-Voltage Spatial Light Modulator by Use of Ultrahigh-Purity GaAs. Japanese Journal of Applied Physics, 49, 102201-102205. http://dx.doi.org/10.1143/JJAP.49.102201

[5] Tharmalingam, M. (1963) Optical Absorption in the Presence of a Uniform Field. Physical Review, 130, $2204-2206$. http://dx.doi.org/10.1103/PhysRev.130.2204

[6] Moss, T.S. (1961) Optical absorption edge in GaAs and Its Dependence on Electric field. Journal of Applied Physics, 32, 2136-2139. http://dx.doi.org/10.1063/1.1777031

[7] Bottka, N. and Hutcheson L.D. (1975) Analytical Aspects of Electroabsorption Modulators. Journal of Applied Physics, 46, 2645-2649. http://dx.doi.org/10.1063/1.321897

[8] Bottka, N. (1978) Materials and Their Properties as They Apply to Electroabsorptive Devices. Optical Engineering, 17, 530-538. http://dx.doi.org/10.1117/12.7972273

[9] Casey Jr., H.C., Sel, D.D. and Wecht, K.W. (1975) Concentration Dependence of the Absorption Coefficient for n- and p-Type GaAs between 1.3 and 1.6 eV. Journal of Applied Physics, 46, 250-257. http://dx.doi.org/10.1063/1.321330

[10] Dow, J.D. and Redfield D. (1970) Electroabsorption in Semiconductors: The Excitonic Absorption Edge. Physical Review B, 1, 3358-3371. http://dx.doi.org/10.1103/PhysRevB.1.3358

[11] Sapkota, D.P., Kayastha, M.S., Takahashi, M. and Wakita, K. (2012) Experimental and Theoretical Study of Excitonic Electroabsorption in High Purity GaAs at Room Temperature. Photonic Global Conference (PGC), 1-4.

[12] Kayastha, M.S., Sapkota, D.P., Takahashi, M. and Wakita, K. (2013) Effect of Electric Field on Exciton in High Purity GaAs Epilayer Measured at Room Temperature. Electronics Letters, 49, 57-59. http://dx.doi.org/10.1049/el.2012.3728

[13] Lin, E.Y., Lay, T.S. and Chang, T.Y. (2007) Accurate Model Including Coulomb-Enhanced and Urbach-Broadened Absorption Spectrum of Direct-Gap Semiconductors. Journal of Applied Physics, 102, 123511-1-123511-10. http://dx.doi.org/10.1063/1.2821361

[14] Schultheis, L. and Kohler, K. (1987) Energy Shift and Line Broadening of Three-Dimensional Excitons in Electric fields. Physical Review B, 36, 6609-6612. http://dx.doi.org/10.1103/PhysRevB.36.6609

[15] Schultheis, L. and Balslev, I. (1983) Excitonic Reflectance of GaAs Crystals Cleaved in Liquid Helium. Physical Review B, 28, 2292-2295. http://dx.doi.org/10.1103/PhysRevB.28.2292 